\title{
Hereditary Angioedema: A Case with Ascites Yet No Symptoms in the Family
}

\author{
Takashi Shinzato, Hiroshi Nakamura, Takao Kuniyoshi, Atsushi Higashionna, Takeshi Uehara, \\ Junichi Oshiro, Hiroyuki Uechi, Koushin ShikiYa, Hiroshi Sakugawa, \\ Fukunori KInjo and Atsushi SaITo
}

\begin{abstract}
An interesting case of hereditary angioedema in a 26-year-old female is reported, with a finding of transient effusion of fluid into the peritoneal cavity during the attacks. The patient suffered from recurrent abdominal pain for several years, but no family members had any similar symptoms. In spite of repeated hospital admissions and many examinations, accurate diagnosis was not made until the most recent admission. The recognition of hereditary angioedema as a cause of acute and/or recurrent abdominal pain may avoid useless invasive procedures and lead to adequate treatment in other similar cases.

(Internal Medicine 31: 633-635, 1992)
\end{abstract}

Key words: C1-esterase inhibitor, recurrent abdominal pain

\section{Introduction}

The evaluation of recurrent abdominal pain is frequently problematic and may lead to invasive procedures $(1,2)$. Although hereditary angioedema (HAE) may present as an abdominal emergency or recurrent abdominal pain (3), HAE is seldom considered in the differential diagnosis. It is a rare disorder and only about 20 families with HAE have been reported in Japan (4). Furthermore, there have been very few reports with fluid effusion into the peritoneal cavity. Here, we present a case of HAE with recurrent abdominal pain and ascites yet no symptoms among family members.

\section{Case Report}

A 26-year-old female had been well until the age of 14 , when she began to experience attacks of painless swelling of her hands and feet which lasted a few days. These attacks occurred sporadically until the age of 16 . At the age of 24 she first began to experience periods of abdominal discomfort when fatigued. At last she was admitted to a hospital for crampy abdominal pain, nausea and vomiting, which diminished gradually within a few days. After that the episodes continued requiring two subsequent admissions. Clinical examination, in- cluding laparoscopy, revealed effusion of straw-colored fluid into the peritoneal cavity (Table 1), and edematously thickened wall of the small bowel. Laboratory data showed no significant findings except a low level of $\mathrm{C} 4$ $(4 \mathrm{mg} / \mathrm{dl})$ and transient hemoconcentration during the attacks. In every episode the ascites and the swelling of the bowel wall disappeared with the diminution of the symptoms. However, the diagnosis was not fully appreciated at the time. In November 1989 at the age of 26 , she suffered from a similar attack with dyspnea. She was referred to our hospital the next day, by which time the symptoms had diminished. She was admitted to our hospital seven days later for further tests.

Her physical examination on admission revealed nothing significant and the laboratory data were within normal limits except for a low $\mathrm{C} 4$ level $(11 \mathrm{mg} / \mathrm{dl})$. That night she suffered another attack with abdominal pain, nausea and vomiting. Physical examination during the attack revealed mild abdominal distension and midabdominal tenderness. Bowel sounds were increased. Her complete blood count at that time showed high values of both white and red blood cell counts $\left(13,600 / \mathrm{mm}^{3}\right.$ and $556 \times 10^{4} / \mathrm{mm}^{3}$ respectively), the hematocrits being $50.7 \%$, while CRP value was $0.74 \mathrm{mg} / \mathrm{dl}$. Plain abdominal radiography showed no findings suggesting ileus. Ultrasonography showed ascites in the peritoneal cavity (Fig.

From the First Department of Internal Medicine, Faculty of Medicine, University of the Ryukyus, Okinawa

Received for publication July 31, 1991; Accepted for publication December 26, 1991

Reprint requests should be addressed to Dr. Takashi Shinzato, the First Department of Internal Medicine, Faculty of Medicine, University of the Ryukyus, 207 Uehara Nishihara-cho, Okinawa 903-01, Japan 


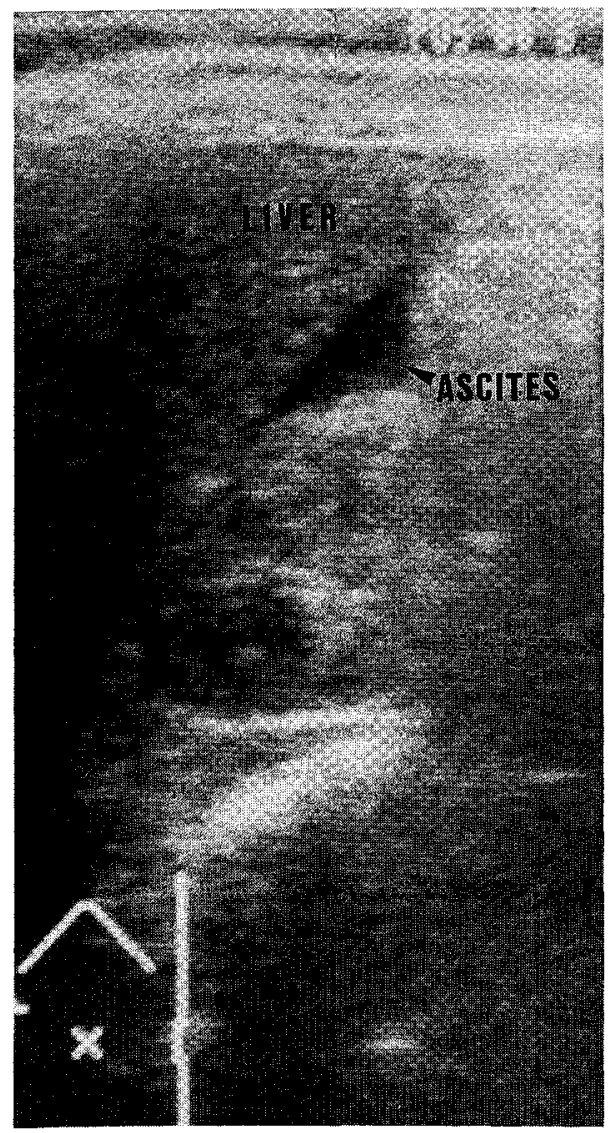

Fig. 1. Ultrasonography showing effusion of fluid into the peritoneal cavity.

1) and swelling of the gastrointestinal wall (Fig. 2), but nothing else significant. Although no medication was administered, the symptoms including the effusion of ascites gradually diminished the next day. Barium radiographs of the small bowel and colon three days

Table 1. Analysis of the Fluid Collected from the Peritoneal Cavity

\begin{tabular}{ll}
\hline Contents & \\
\hline Appearance & Clear, straw-colored \\
Specific gravity & 1.034 \\
Protcin content & $4.8 \mathrm{~g} / \mathrm{dl}$ \\
Red blood cell & $1+$ \\
White cell count & $100 / \mathrm{mm}^{3}$ \\
& (Eo 3\%, Neu 8, Ly 3, \\
& Mono 86) \\
Amylase & $41 \mathrm{IU} / \mathrm{1}$ \\
LDH & $114 \mathrm{IU} / \mathrm{l}$ \\
Glucose & $107 \mathrm{mg} / \mathrm{dl}$ \\
Culture for bacteria & Negative \\
Cytology & No malignant cells
\end{tabular}

Eo: cosinophils, Ncu: neutrophils, LY: lymphocytes, Mono: monocytes.

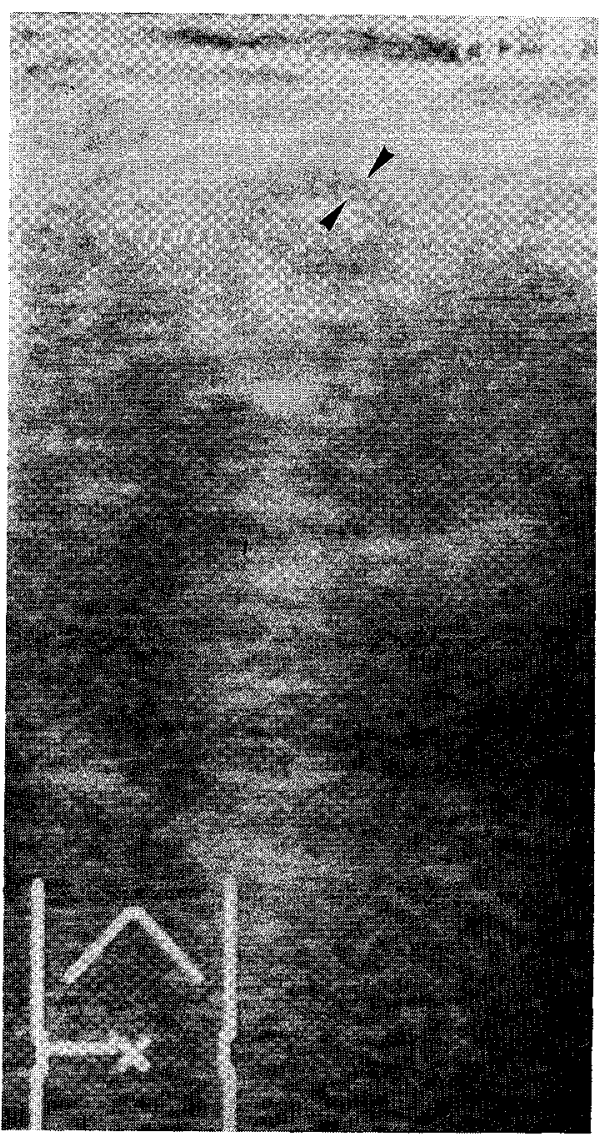

Fig. 2. Ultrasonography at the time of the attack showing swelling of the bowel wall: $6 \mathrm{~mm}$ thick (arrows).

later showed no abnormal signs. Infectious diseases due to parasites or viruses, collagen diseases and malignancies were ruled out by the absence of antinuclear antibodies or parasite eggs in feces, and by normal levels of both serum immunoglobulin and tumor markers. Gynecological disorders were also ruled out by examination. Angioedema was suspected, and complement activity was measured five days later when the symptoms had diminished. Low levels of $\mathrm{C} 4, \mathrm{C} 1 \mathrm{INH}$ and $\mathrm{C} 1 \mathrm{INH}$

Table 2. Data of the Patient and Members of Her Family, Showing Abnormal Complement Activity

\begin{tabular}{lrlcccc}
\hline Subject & $\begin{array}{l}\text { Age Symptoms } \\
(\mathrm{yr})\end{array}$ & $\begin{array}{c}\mathrm{C} 3 \\
60-120^{*}\end{array}$ & $\begin{array}{c}\mathrm{C} 4 \\
24-46^{*}\end{array}$ & $\begin{array}{c}\mathrm{C} 1 \mathrm{INH} \\
15-35^{*}\end{array}$ & $\begin{array}{c}\mathrm{C} 1 \mathrm{INH} \\
\text { activity } \\
80-120 \%\end{array}$ \\
\hline Patient & 26 & $\mathrm{AP}, \mathrm{N}, \mathrm{V}$ & 73 & 11 & 5 & 17 \\
Father & 51 & None & 42 & 11 & 5 & 18 \\
Brother & 28 & None & 75 & 11 & 7 & 22 \\
Son & 3 & None & 47 & 11 & 15 & $\mathrm{NT}$ \\
Father's sister & 76 & None & 66 & 14 & 33 & 86 \\
\hline
\end{tabular}

AP: abdominal paint, $\mathrm{N}$ : nausea, $\mathrm{V}$ : vomiting, INH: esterase inhibitor, NT: not tested.

* normal range in $\mathrm{mg} / \mathrm{dl}$. 
activity confirmed the diagnosis of HAE (Table 2).

Having established the diagnosis, the patient's family were interviewed and their serum $\mathrm{C} 1 \mathrm{INH}, \mathrm{Cl} \mathrm{INH}$ activity, C3 and C4 levels were measured. None of the examined family members, her father, mother, son, one of her brothers and one of her aunts, had ever experienced any symptoms of HAE. However, laboratory data demonstrated an abnormally low value of $\mathrm{C} 1 \mathrm{INH}$ activity in both her father and brother, and low $\mathrm{C} 4$ and $\mathrm{C} 1 \mathrm{INH}$ values in the son, suggesting subclinical HAE (Table 2).

\section{Discussion}

HAE is a rare autosomal dominant disorder that results from a deficiency or dysfunction of C1 INH $(5,6)$. This disorder is characterized by recurrent episodes of soft tissue swelling, particularly skin and mucous membranes, especially those lining the pharynx, larynx and gastrointestinal tract, and by the onset of symptoms in childhood or early adolescence $(6,7)$. Often, the onset of the episodic attack is associated with stress or trauma, infection, or changes in temperature or sex hormone levels. The clinical diagnosis can be confirmed by findings of low levels of $\mathrm{C} 4$ and $\mathrm{C} 1 \mathrm{INH}$ or absent $\mathrm{C} 1$ INH functional activity, or by the presence of a positive family history (6).

Gastrointestinal involvement, such as crampy abdominal pain or small bowel obstruction, may be sometimes the first or only presenting symptom $(2,3)$. Until diagnosed, the abdominal pain may often lead to repeated hospital admissions, surgery or labeling the patient as neurotic or hysterical (8). The present case seems to be typical in these respects.

The pathogenesis of edema formation is not clearly understood. The reduced levels of functional $\mathrm{C} 1 \mathrm{INH}$ can lead to uncontrolled activation of the classical pathway of complement (6). This may give rise to the release of kinin-like substances that have been assumed to cause increased capillary permeability $(6,7)$, and increased blood concentration and transient increased neutrophils may follow that (9). The isolation, structure and origin of the permeability factors remain to be definitely established (6). The fact that the edema dramatically disappears following the administration of $\mathrm{C} 1 \mathrm{INH}$ concentrates $(10-12)$ suggests that the main cause of this disorder is C1 INH deficiency or dysfunction, whatever factor directly causes the edema.

Few reports of HAE have referred to the effusion of fluid into the peritoneal cavity. According to the mechanism described above, extravasation of intravascular fluid and protein into submucosal structures may be responsible for the effusion of fluid. Pearson et al pointed out the rapid and complete reversibility of the gastrointestinal roentgenographic findings in HAE (13). The reversibility is considered to be caused by the same mechanism as the ascites. Therefore transient signs of ascites with unexplained recurrent abdominal pain would be findings suggestive of HAE.

In patients with HAE, long-term prophylaxis with danazol, stanozolol, epsilonaminocaproic acid and tranexamic acid is usually effective $(6,7)$. It is necessary in acute attacks to perform replacement therapy with a concentrate of purified $\mathrm{C} 1 \mathrm{INH}$ or with fresh, frozen plasma $(6,7)$, because the occurrence of laryngeal edema sometimes leads to sudden death. The mortality rate of this disorder is as high as $30 \%$; It is notable, though, that the mortality rate is decreasing with better diagnosis and therapy (6).

In the present patient, the gastrointestinal symptoms were the conspicuous manifestations of HAE during the attacks, but the family had no history of such symptoms. The recognition or awareness of HAE as a cause of acute and/or recurrent abdominal pain is important, and may at least avoid unnecessary invasive procedures and facilitate adequate treatment. For this reason HAE should be included in the differential diagnosis for patients with these symptoms.

\section{References}

1) Way LW. Abdominal pain. In: Gastrointestinal Disease, Sleisenger MH, Fordtran JS, Eds. WB Saunders, Philadelphia, 1983, p. 203.

2) Weinstock LB, Kothari T, Sharma RN, Rosenfeld SI. Recurrent abdominal pain as the sole manifestation of hereditary angioedema in multiple family members. Gastroenterology 93: 1116, 1987.

3) Warin RP, Higgs ER. Acute and recurrent abdominal pain due to hereditary angioedema. Br Med J [Clin Res] 1912: 284, 1982.

4) Nagaki K. Hereditary angioedema. Taisya 21: 261, 1984 (in Japanese).

5) Donaldson VH, Evans RR. A biochemical abnormality in hereditary angioneurotic edema. Am J Med 35: 37, 1963.

6) Frank MD, Gelfand JA, Atkinson JP. Hereditary angioedema: clinical syndrome and its management. Ann Intern Med 84: $580,1976$.

7) Bork K, Witzke G. Long-term prophylaxis with C1-inhibitor concentrate in patients with recurrent angioedema caused by hereditary and acquired C1-inhibitor deficiency. J Allergy Clin Immunol 83: 677, 1989.

8) Collen MJ, Lewis JH, Deschner WK, et al. Abdominal pain in hereditary angioedema: the role of acid hypersecretion. Am J Gastroenterol 84: 873, 1989.

9) Kodama J. The pathogenicity and treatment of hereditary angioedema. Chiryo 67: 1415, 1985 (in Japanese).

10) Gadek JE, Hosea SW, Gelfand JA, et al. Replacement therapy in hereditary angioedema. N Engl J Med 6: 542, 1980.

11) Kodama J, Uchida K, Yoshimura S, et al. Studies of four Japanese families with hereditary angioneurotic edema: simultaneous activation of plasma protease systems and exogenous triggering stimuli. Blut 49: 405, 1984.

12) Kodama J, Uchida K, Nakayama R. Clinical effect of C1-inhibitor concentrate in patients with hereditary angioneurotic edema. Shinyaku To Chiryo 24: 2041, 1987 (in Japanese).

13) Pearson KD, Buchignani JS, Shimkin PM, Frank MM. Hereditary angioeurotic edema of the gastrointestinal tract. Am J Roentgenol 116: $256,1972$. 